TRANSACTIONS OF THE

AMERICAN MATHEMATICAL SOCIETY

Volume 357, Number 5, Pages 2007-2020

S 0002-9947(04)03581-0

Article electronically published on October 7, 2004

\title{
CUT NUMBERS OF 3-MANIFOLDS
}

\author{
ADAM S. SIKORA
}

\begin{abstract}
We investigate the relations between the cut number, $c(M)$, and the first Betti number, $b_{1}(M)$, of 3-manifolds $M$. We prove that the cut number of a "generic" 3-manifold $M$ is at most 2 . This is a rather unexpected result since specific examples of 3-manifolds with large $b_{1}(M)$ and $c(M) \leq 2$ are hard to construct. We also prove that for any complex semisimple Lie algebra $\mathfrak{g}$ there exists a 3 -manifold $M$ with $b_{1}(M)=\operatorname{dim} \mathfrak{g}$ and $c(M) \leq \operatorname{rank} \mathfrak{g}$. Such manifolds can be explicitly constructed.
\end{abstract}

\section{INTRODUCTION}

Let $M$ be a closed, smooth manifold. The cut number of $M, c(M)$, is the largest number of disjoint two-sided hypersurfaces $\mathcal{F}_{1}, \ldots, \mathcal{F}_{c}$, which do not separate $M$, i.e. surfaces such that $M \backslash\left(\mathcal{F}_{1} \cup \ldots \cup \mathcal{F}_{c}\right)$ is connected.

By [St, Prop. 4.2] (see also our Proposition [3), $c(M)$ is the corank of $\pi_{1}(M)$. The corank of a group $\Gamma$ is the largest number $n$ for which the free group on $n$ generators, $F_{n}$, is an epimorphic image of $\pi_{1}(M)$.

Although the corank of a group is not a very tangible quantity, it can be calculated (at least theoretically) for any finitely presented group. This calculation reduces to finding the ranks of solutions of certain systems of equations on free groups. The algorithm for finding such ranks was given in [Ra, §9].

Since $c(M)$ is bounded from above by the first Betti number of $M, b_{1}(M)$, it is natural to ask whether there exists an analogous lower bound on $c(M)$. In particular, T. Kerler and J. H. Przytycki asked whether

$$
\frac{1}{3} b_{1}(M) \leq c(M)
$$

for any closed, oriented 3-manifold $M$. This question was motivated by the following two inequalities:

$$
\frac{1}{3} b_{1}(M) \leq \mathcal{O}_{p}(M) / \mathcal{O}_{p}\left(S^{2} \times S^{1}\right)
$$

and

$$
c(M) \leq \mathcal{O}_{p}(M) / \mathcal{O}_{p}\left(S^{2} \times S^{1}\right), \text { for } p=5,
$$

proved by Cochran and Melvin [CM, Thm 4.3], and Gilmer and Kerler, respectively. Here $\mathcal{O}_{p}(M)$ denotes the quantum order of $M$, that is the largest $k$ such that $\tau_{p}(M) /\left(\xi_{p}-1\right)^{k} \in \mathbb{Z}\left[\xi_{p}\right]$, where $\xi_{p}=e^{2 \pi i / p}$ and $\tau_{p}(M)$ is the $S O(3)$-quantum invariant of 3-manifolds. (We assume that $\tau_{p}\left(S^{3}\right)=1$.) Note that (1) holds for

Received by the editors October 28, 2002 and, in revised form, December 2, 2003.

2000 Mathematics Subject Classification. Primary 57M05, 57M27, 20F34, 11E76.

Key words and phrases. Cut number, 3-manifold, corank, skew-symmetric form, cohomology ring. 
(a) Seifert 3-manifolds, (b) 3-manifolds with abelian fundamental groups, (c) 3manifolds $M$ with $b_{1}(M) \leq 3$. Furthermore, for closed surfaces $\mathcal{F}, \frac{1}{2} b_{1}(\mathcal{F})=c(\mathcal{F})$.

We are going to prove the following two results contradicting (11):

Theorem 1. For any semisimple Lie algebra $\mathfrak{g}$ over $\mathbb{C}$ there exists a closed, oriented 3-manifold $M$ with $b_{1}(M)=\operatorname{dim} \mathfrak{g}$ and $c(M) \leq \operatorname{rank} \mathfrak{g}$.

The proof and information on how to construct such 3-manifolds can be found in Section 3 .

The dimensions of simple Lie algebras of rank $r$ are as follows:

$$
\begin{gathered}
\operatorname{dim} s l_{r+1}=r(r+2), \quad \operatorname{dim} s o_{2 r+1}=\operatorname{dimsp}_{2 r}=r(2 r+1), \quad \operatorname{dim} s o_{2 r}=r(2 r-1), \\
\operatorname{dim} G_{2}=14, \operatorname{dim} F_{4}=52, \operatorname{dim} E_{6}=78, \operatorname{dim} E_{7}=133, \operatorname{dim} E_{8}=248 .
\end{gathered}
$$

Therefore, Theorem 11 provides many counterexamples for (11). In particular, it implies the existence of 3-manifolds with $b_{1}(M)=8,10,14$ and $c(M) \leq 2$.

Let $\Omega_{\mathbb{Q}}(n)$ be the space of all skew-symmetric 3-forms on $\mathbb{Q}^{n}, \Omega_{\mathbb{Q}}(n)=\left(\bigwedge^{3} \mathbb{Q}^{n}\right)^{*}$. For a given closed, oriented 3-manifold $M$, a choice of a basis of $H^{1}(M, \mathbb{Q})$ provides an isomorphism $H^{1}(M, \mathbb{Q})=\mathbb{Q}^{n}$ for $n=b_{1}(M)$ and makes it possible to consider the cup product 3 -form on $H^{1}(M, Q), \Psi_{M}(x, y, z)=(x \cup y \cup z)[M]$, as an element of $\Omega_{\mathbb{Q}}(n)$.

The cup 3-form, $\Psi_{M}$, determines the rational cohomology ring of $M$. Since by a theorem of Sullivan, $\underline{\mathrm{Su}}$. (compare also $[\mathrm{T2}]$ ), every 3 -form corresponds to the cup product form for some closed oriented 3-manifold, $\Omega_{\mathbb{Q}}(n)$ can be considered as a space classifying the rational cohomology rings of 3-manifolds $M$ with a specified basis of $H^{1}(M, \mathbb{Q})$.

Theorem 2. (1) The set of forms $\Psi_{M} \in \Omega_{\mathbb{Q}}(n)$ corresponding to 3-manifolds $M$ with $c(M) \leq 2$ contains an open, dense subset (in the Zariski and Euclidean topologies) of $\Omega_{\mathbb{Q}}(n)$.

(2) In particular, for any $n$ there exists a 3-manifold $M$ with $b_{1}(M)=n$ and $c(M) \leq 2$.

(3) For any $n \geq 9$ there exist infinitely many 3 -manifolds $M$ with $b_{1}(M)=n$, $c(M) \leq 2$, and with pairwise non-isomorphic rational cohomology rings 1

Part (1) of the above result implies that the cut number of a "generic" 3-manifold is at most 2 . This is a rather surprising result, since it is not easy to construct specific examples of 3-manifolds with large $b_{1}(M)$ and $c(M) \leq 2$.

In an independent work on Kerler's and Przytycki's question, S. Harvey found an explicit family of 3-manifolds $M_{n}$ with $b_{1}\left(M_{n}\right)=n$ and $c\left(M_{n}\right)=1$, $\mathrm{Har}$. 2 For the purpose of her construction, one takes a link $L_{n} \subset S^{3}$ of $n$ components, obtained from the unlink by linking every pair of components in the Whitehead link manner. Harvey's manifold $M_{n}$ is the result of the 0-surgery on $L_{n}$.

In yet another independent work inspired by the same problem, C. Leininger and A. Reid showed that there exist examples of a hyperbolic 3-manifolds with $c(M)=1$ and $b_{1}(M)=5,[\mathrm{LR}]$.

\footnotetext{
${ }^{1}$ The assumption $n \geq 9$ is related to the fact that for any $n \leq 8$ the cohomology rings, $H^{*}(M, \mathbb{C})$, of 3 -manifolds $M$ with $b_{1}(M)=n$ belong to a finite number of isomorphism classes only.

${ }^{2}$ Both S. Harvey and I announced our results in September 2001.
} 


\section{Cut Number AND ITS SIBLings}

We will denote the corank of $\Gamma$ by $d(\Gamma)$.

Proposition 3 (compare St, Prop. 4.2]). For a closed, smooth manifold $M$, $c(M)=d\left(\pi_{1}(M)\right)$.

Proof. Any epimorphism $\pi_{1}(M) \rightarrow F_{c}$ induces a map $f: M \rightarrow \bigvee_{1}^{c} S^{1}$ which can be assumed smooth outside the preimage of the base point. If $y_{1}, \ldots, y_{c}$ are regular values of $f$ lying in different circles, then $f^{-1}\left(y_{1}\right), \ldots, f^{-1}\left(y_{c}\right)$ are disjoint, twosided surfaces in $M$. Since these surfaces represent $c$ linearly independent elements of $H_{2}(M, \mathbb{Z} / 2)$, for each $i$ one can choose a connected component $\mathcal{F}_{i} \subset f^{-1}\left(y_{i}\right)$ such that $\mathcal{F}_{1}, \ldots, \mathcal{F}_{c}$ are linearly independent in $H_{2}(M, \mathbb{Z} / 2)$ as well. The following lemma shows that $c(M) \geq d\left(\pi_{1}(M)\right)$.

Lemma 4. $\mathcal{F}_{1}, \ldots, \mathcal{F}_{c}$ do not separate $M$.

Proof. Let loops $\gamma_{1}, \ldots, \gamma_{c}$ represent elements of $H_{1}(M, \mathbb{Z} / 2)$ dual to $\mathcal{F}_{1}, \ldots, \mathcal{F}_{c}, \gamma_{i}$. $\mathcal{F}_{j}=\delta_{i j} \bmod 2$. Assume that $\gamma_{i}$ intersects $\mathcal{F}_{j}$ in at least two points $p_{1}, p_{2}$. Let $C_{1}, C_{2}$ be $\operatorname{arcs}$ connecting $p_{1}$ and $p_{2}$, such that $C_{1}$ and $C_{2}$ lie on different sides of $\mathcal{F}_{j}$ and do not intersect other surfaces. By cutting $\gamma_{i}$ at $p_{1}$ and $p_{2}$ and inserting the arcs $C_{1}$ and $C_{2}$ into $\gamma_{i}$ we obtain a new curve with two less intersections with $\mathcal{F}_{j}$ and the number of intersections with $\mathcal{F}_{j^{\prime}}$ unchanged for $j^{\prime} \neq j$. By applying this operation as many times as necessary, we get closed curves $\gamma_{1}^{\prime}, \ldots, \gamma_{c}^{\prime}$ such that $\gamma_{i}^{\prime}$ intersects $\mathcal{F}_{i}$ only once and it does not intersect $\mathcal{F}_{j}$ for $j \neq i$. Consider a path $\gamma$ in $M$ joining two arbitrary points $x$ and $y$ in $M \backslash\left(\mathcal{F}_{1}, \ldots, \mathcal{F}_{c}\right)$. By by deforming $\gamma$ appropriately and pasting $\gamma \cdot \mathcal{F}_{i}$ copies of $\gamma_{i}^{\prime}$ into $\gamma$ we obtain a new path connecting $x$ and $y$ which does not intersect $\mathcal{F}_{1}, \ldots, \mathcal{F}_{c}$.

We continue proving Proposition 3 Let $\mathcal{F}_{1}, \ldots, \mathcal{F}_{c}$ be disjoint, two-sided surfaces which do not separate $M$ and let $N_{1}, . ., N_{c}$ be disjoint neighborhoods of $\mathcal{F}_{1}, \ldots, \mathcal{F}_{c}$ provided with parameterizations $N_{i} \stackrel{\pi_{i} \times \lambda_{i}}{\longrightarrow}[-1,1] \times \mathcal{F}_{i}$. Consider the map $f: M \rightarrow$ $\bigvee_{1}^{c} S^{1}$ composed of the constant map $M \backslash\left(N_{1} \cup \ldots \cup N_{c}\right) \rightarrow *$ and the maps $N_{i} \stackrel{\pi_{i}}{\rightarrow}$ $[-1,1] \rightarrow S^{1}$, where $S^{1}$, obtained by identifying the end points of $[-1,1]$, is the $i$-th circle in $\bigvee_{1}^{c} S^{1}$. Since $f$ induces an epimorphism $f_{*}: \pi_{1}(M) \rightarrow F_{c}, d\left(\pi_{1}(M)\right) \geq$ $c(M)$.

The cut number can be also defined for simplicial complexes:

Definition 5. For any simplicial complex $X$ define $c(X)$ to be the largest number $n$ such that there exists a subdivision $X^{\prime}$ of $X$ and 1-cocycles $\alpha^{1}, \ldots, \alpha^{n} \in C^{1}\left(X^{\prime}\right)$ such that $\alpha^{i} \cup \alpha^{j}=0$ in $C^{2}\left(X^{\prime}\right)$ for all $i \neq j$, and such that $\left[\alpha^{1}\right], \ldots,\left[\alpha^{n}\right] \in H^{1}(X, \mathbb{Z})$ are linearly independent.

Above, we assume that $X^{\prime}$ is an ordered simplicial complex and the cup product on $C^{1}\left(X^{\prime}\right)$ is given by the Alexander-Whitney diagonal approximation

$$
\left.(\alpha \cup \beta)\left\langle x_{0}, x_{1}, x_{2}\right\rangle=-\alpha\left(\left\langle x_{0}, x_{1}\right\rangle\right) \beta\left\langle x_{1}, x_{2}\right\rangle\right) ;
$$

compare Bre VI§4].

The following generalizes Proposition 4.4 in $[\mathrm{Dw}]$.

Proposition 6. $c(X)=d\left(\pi_{1}(X)\right)$ for any simplicial complex $X$. 
Proof. If $d\left(\pi_{1}(X)\right)=n$, then there exists a simplicial subdivision $X^{\prime}$ of $X$ and a triangulation of $\bigvee_{1}^{n} S^{1}$, such that there exists a simplicial map $f: X \rightarrow \bigvee_{1}^{n} S^{1}$ inducing an epimorphism $\pi_{1}(X) \rightarrow \pi_{1}\left(\bigvee_{1}^{n} S^{1}\right)$. Let $\beta_{i} \in C^{1}\left(\bigvee_{1}^{n} S^{1}\right)$ be a cocycle assigning 1 to a certain 1-simplex lying in the $i$ th circle and 0 to all other simplices of $\bigvee_{1}^{n} S^{1}$. Then $\alpha_{i}=f^{*}\left(\beta_{i}\right) \in C^{1}\left(X^{\prime}\right)$, for $i=1, \ldots, n$, satisfy the conditions of the definition of $c(X)$ and, hence, $c(X) \geq d\left(\pi_{1}(X)\right)$.

Conversely, let $\alpha_{1}, \ldots, \alpha_{n} \in C^{1}\left(X^{\prime}\right)$ satisfy the conditions of the definition of $c(X)$. Consider the map $f:\left(X^{\prime}\right)^{1} \rightarrow \bigvee_{1}^{n} S^{1}$ which maps all vertices of $X^{\prime}$ to the base point of $\bigvee_{1}^{n} S^{1}$ and any 1-simplex $v$ to a curve $f(v)$ in $\bigvee_{1}^{n} S^{1}$ going $\alpha_{1}(v)$ times around the first circle, $\alpha_{2}(v)$ times around the second, and so on (in that order).

Since $\alpha_{i} \cup \alpha_{j}=0$ in $C^{2}\left(X^{\prime}\right)$ for $i \neq j$ for any 2-simplex $\left\langle x_{0}, x_{1}, x_{2}\right\rangle$ in $X^{\prime}$ one of the following 3 conditions holds: either $\alpha_{i}\left(\left\langle x_{0}, x_{1}\right\rangle\right)=\alpha_{i}\left(\left\langle x_{1}, x_{2}\right\rangle\right)=0$ for all $i$ different than a certain $k$, or $\alpha_{i}\left(\left\langle x_{0}, x_{1}\right\rangle\right)=0$ for all $i$ or $\alpha_{i}\left(\left\langle x_{1}, x_{2}\right\rangle\right)=0$ for all $i$. In each of these cases $f$ extends onto $\left\langle x_{0}, x_{1}, x_{2}\right\rangle$. Hence, $f$ extends onto the 2-skeleton, $\left(X^{\prime}\right)^{2}$, and consequently it extends to a map $f: X^{\prime} \rightarrow \bigvee_{1}^{n} S^{1}$. Since $\left[\alpha_{1}\right], \ldots,\left[\alpha_{n}\right] \in H^{1}(X, \mathbb{Z})$ are linearly independent, the image of the map $f_{*}: H_{1}(X, \mathbb{Z}) \rightarrow H_{1}\left(\bigvee_{1}^{n} S^{1}\right)$ is isomorphic with $\mathbb{Z}^{n}$. Therefore the image of $f_{*}$ : $\pi_{1}(X) \rightarrow \pi_{1}\left(\bigvee_{1}^{n} S^{1}\right)$ is the free group of rank $n$. Hence $d\left(\pi_{1}(X)\right) \geq c(X)$.

We will say that a set of vectors $v_{1}, \ldots, v_{n} \in H^{1}(X, \mathbb{Z})$ is primitive if the map $\left(v_{1}, \ldots, v_{n}\right): H_{1}(X, \mathbb{Z}) \rightarrow \mathbb{Z}^{n}$ is an epimorphism. Primitive vectors are linearly independent. For any topological space $X$, let $c_{k}(X)$ for $k=1,2, \ldots$, be the cardinality of the largest set of primitive vectors $v_{1}, \ldots, v_{n} \in H^{1}(X, \mathbb{Z})$ such that the all Massey products $\left\langle v_{i_{1}}, \ldots, v_{i_{l}}\right\rangle$ for $l \leq k$ are uniquely defined and equal to 0 . The definition of the Massey product can be found for example in [Fe. In particular, $c_{2}(X)$ is the cardinality of the largest primitive set of vectors $v_{1}, \ldots, v_{n} \in H^{1}(X, \mathbb{Z})$ such that $v_{i} \cup v_{j}=0$ for all $i, j$. We will call $c_{2}(X)$ the algebraic cut number of $X$.

If $\alpha_{1}, \ldots, \alpha_{n} \in C^{1}(X)$ are as in Definition $\left[5\right.$ then all Massey products $\left\langle\left[\alpha_{i_{1}}\right], \ldots\right.$, $\left.\left[\alpha_{i_{k}}\right]\right\rangle$ are 0 . For that reason we introduce the notation $c_{\infty}(X)=c(X)$. We will see in Corollary 11 that $c_{\infty}(X) \leq c_{k}(X)$ for any $k$ and therefore

$$
c_{\infty}(X) \leq \ldots \leq c_{k}(X) \leq \ldots \leq c_{2}(X) \leq c_{1}(X)=b_{1}(X) .
$$

Notice that the numbers $c_{k}(X)$ depend on the homotopy type of $X$ only 3 Furthermore, the next proposition shows that these numbers can be derived from $\pi_{1}(X)$. Let $K(\Gamma, 1)$ denote the Eilenberg-Mac Lane space for $\Gamma$ and let $c_{k}(\Gamma)=c_{k}(K(\Gamma, 1))$.

Proposition 7. If $X$ is a $C W$-complex, then $c_{k}(X)=c_{k}\left(\pi_{1}(X)\right)$ for all $k$ 青

Proof. By Proposition 6, for $k=\infty$ we have $c_{\infty}(X)=d\left(\pi_{1}(X)\right)=c_{\infty}\left(K\left(\pi_{1}(X), 1\right)\right)$. Hence, we may assume that $k<\infty$. The space $K\left(\pi_{1}(X), 1\right)$ can be constructed from $X$ by attaching $n$-cells, $n \geq 3$. Therefore the natural map $\psi: X \rightarrow K\left(\pi_{1}(X), 1\right)$ yields a one-to-one correspondence between the $i$-cells of $X$ and the $i$-cells of $K\left(\pi_{1}(X), 1\right)$ for $i=0,1,2$ and it induces an isomorphism $H^{1}\left(K\left(\pi_{1}(X), 1\right), \mathbb{Z}\right) \rightarrow$ $H^{1}(X, \mathbb{Z})$ and a monomorphism $H^{2}\left(K\left(\pi_{1}(X), 1\right), \mathbb{Z}\right) \rightarrow H^{2}(X, \mathbb{Z})$. Now the statement follows directly from the definition of the Massey product (defined for cell cohomology).

\footnotetext{
${ }^{3}$ Here one uses [Fe Lemma 6.2.5].

${ }^{4}$ Because of the assumption of Definition [5 for $k=\infty$ we assume that $X$ is a simplicial complex.
} 
Proposition 8. If $G_{1}$ is an epimorphic image of $G_{0}$, then $c_{k}\left(G_{1}\right) \leq c_{k}\left(G_{0}\right)$ for each $k$.

Proof. For $k=\infty$ the statement follows from Proposition [6 and therefore assume that $k<\infty$. Since $G_{1}$ is an epimorphic image of $G_{0}$, the Eilenberg-Mac Lane spaces for $G_{0}$ and $G_{1}$ may be constructed in such a way that there exists a cellular map $f: K\left(G_{0}, 1\right) \rightarrow K\left(G_{1}, 1\right)$ which is a bijection between the 1-cells. (Consequently, $f_{*}: G_{0}=\pi_{1}\left(K\left(G_{0}, 1\right)\right) \rightarrow \pi_{1}\left(K\left(G_{1}, 1\right)\right)=G_{1}$ is an epimorphism.) Let $c_{k}\left(G_{1}\right)=n$ and let $\left\{v_{1}, \ldots, v_{n}\right\}$ be a primitive set of elements of $H^{1}\left(G_{1}, \mathbb{Z}\right)$ such that all Massey products $\left\langle v_{i_{1}}, \ldots, v_{i_{k}}\right\rangle$ are 0 . Then $\left\{f^{*}\left(v_{1}\right), \ldots, f^{*}\left(v_{n}\right)\right\}$ is a primitive set of elements of $H^{1}\left(G_{0}, \mathbb{Z}\right)$ and we claim that all Massey products $\left\langle f^{*}\left(v_{i_{1}}\right), \ldots, f^{*}\left(v_{i_{k}}\right)\right\rangle$ are 0 . Indeed, since $f$ is a bijection between $C_{1}\left(K\left(G_{0}, 1\right)\right)$ and $C_{1}\left(K\left(G_{1}, 1\right)\right)$ any defining set for the Massey product $\left\langle f^{*}\left(v_{i_{1}}\right), \ldots, f^{*}\left(v_{i_{k}}\right)\right\rangle$ is of the form $\left(f^{*}\left(a_{i j}\right)\right)_{i, j}$ where $\left(a_{i j}\right)_{i, j}$ is a defining set for $\left\{v_{1}, \ldots, v_{n}\right\}$. Hence $\left\langle f^{*}\left(v_{i_{1}}\right), \ldots, f^{*}\left(v_{i_{k}}\right)\right\rangle=f^{*}\left(\left\langle v_{i_{1}}, \ldots, v_{i_{k}}\right\rangle\right)=0$.

By Proposition [6. $c_{\infty}(\Gamma)=d(\Gamma)$. Therefore, it is natural to ask whether there exists a similar interpretation of the numbers $c_{k}(\Gamma)$ for $k<\infty$. One may consider a sequence of numbers $d_{k}(\Gamma)$ defined as follows: If $F_{n, k}$ denotes the $k$ th group in the lower central series of $F_{n}, F_{n, 0}=F_{n}, F_{n, k+1}=\left[F_{n, k}, F_{n}\right]$, then $d_{k}(\Gamma)$ is the largest $n$ for which there exists an epimorphism from $\Gamma$ onto $F_{n} / F_{n, k}$. Let $d_{\infty}(\Gamma)=d(\Gamma)$. The following sequence of inequalities parallels (2):

$$
d_{\infty}(\Gamma) \leq \ldots \leq d_{k}(\Gamma) \leq \ldots \leq d_{1}(\Gamma) .
$$

Conjecture 1. $c_{k}(\Gamma)=d_{k}(\Gamma)$ for any $k$ 占

Indeed, $c_{k}(\Gamma)=d_{k}(\Gamma)$ for $k=1$ and $k=\infty$. Later on, we will prove it for $k=2$ as well. Furthermore, we have the following inequality:

Proposition 9. $d_{k}(\Gamma) \leq c_{k}(\Gamma)$ for any $k$.

Proof. Since for $k=\infty$ this follows from Proposition [6, assume that $k<\infty$. If $d_{k}(\Gamma)=n$, then $F_{n, k}$ is an epimorphic image of $\Gamma$ and by Proposition $8, c_{k}(\Gamma) \geq$ $c_{k}\left(F_{n, k}\right)$. Now the statement follows from the lemma below.

Lemma 10. $c_{k}\left(F_{n} / F_{n, k}\right)=n$.

Proof. Since $H^{1}\left(F_{n} / F_{n, k}, \mathbb{Z}\right)=\mathbb{Z}^{n}$, it is enough to prove that for all $v_{1}, \ldots, v_{k} \in$ $H^{1}\left(F_{n} / F_{n, k}\right),\left\langle v_{1}, \ldots, v_{k}\right\rangle=0$. Let $U(n) \subset G L(\mathbb{Z}, n)$ be the group of upper triangular $n \times n$ matrices all of whose diagonal entries are 1. By [Dw, Thm 2.4], it is sufficient to prove that for any $v_{1}, \ldots, v_{k}: F_{n} / F_{n, k} \rightarrow \mathbb{Z}$ there exists a homomorphism $f: F_{n} / F_{n, k} \rightarrow U(k+1)$ whose $(i, i+1)$ component is $v_{i}$, for $i=1, \ldots, k$. Since $F_{n}$ is a free group, there exists a homomorphism $\bar{f}: F_{n} \rightarrow U(k+1)$ with the above property. Since the $k$ th commutator of $U(k+1)$ is the trivial group, $\bar{f}$ factors through $F_{n, k}$ yielding the required homomorphism.

\footnotetext{
${ }^{5}$ One needs to show that if $c_{k}(\Gamma)=n$, then there exists an epimorphism $\Gamma \rightarrow F_{n} / F_{n, k}$. Here is one possible approach suggested to us: The Magnus expansion of the free group $F_{n}$ provides an embedding of $F_{n}$ into the ring of formal power series in $n$ non-commuting variables, [MKS]. By taking a quotient of this ring by the ideal generated by all monomials of degree $k$, this map factors to an embedding of $F_{n} / F_{n, k}$ into the ring of polynomials $\sum_{r=0}^{k} \sum_{i_{1}, \ldots, i_{r}} a_{i_{1} \ldots i_{r}} x_{i_{1}} \ldots x_{i_{r}}$, where $a_{i_{1} \ldots i_{r}} \in \mathbb{Z}$ and $x_{1}, \ldots, x_{n}$, are non-commuting variables. Therefore, any epimorphism $\Gamma \rightarrow F_{n} / F_{n, k}$ is determined by the functions $a_{i_{1} \ldots i_{r}}: \Gamma \rightarrow \mathbb{Z}$. Those functions are related to the Massey products for $\Gamma$. In the case of complements of links in homology spheres, this connection is mentioned in [T1].
} 
Since $c_{\infty}(X)=d_{\infty}\left(\pi_{1}(X)\right) \leq d_{k}\left(\pi_{1}(X)\right) \leq c_{k}\left(\pi_{1}(X)\right)$ we get

Corollary 11. For any simplicial complex $X$ and any $k, c_{\infty}(X) \leq c_{k}(X) 6$

Now we are going to prove Conjecture 1 for $k=2$. First, we recall a few results from homological algebra.

Given a group $G$ and an abelian group $A$, the extensions $E$ of $G$ by $A$, given by the following short exact sequence

$$
0 \rightarrow A \rightarrow E \stackrel{\pi}{\rightarrow} G \rightarrow 0
$$

(up to an isomorphism), are in one-to-one correspondence with elements of $H^{2}(G, A)$, where the action of $G$ on $A$ comes from the action of $E$ by conjugation on $A$. We denote the element of $H^{2}(G, A)$ corresponding to such extension by $\xi$. We say that the sequence splits if there exists a homomorphism $\tau: G \rightarrow E$ such that $\pi \tau=i d_{G}$. This happens if and only if $\xi=0$.

Lemma 12. (1) A group homomorphism $f: \Gamma \rightarrow G$ lifts to a homomorphism $\bar{f}: \Gamma \rightarrow E$ if and only if $f^{*}(\xi)=0$ in $H^{2}(\Gamma, A)$.

(2) If $f^{*}(\xi)=0$ and $f$ is an epimorphism, then also $\bar{f}$ is an epimorphism.

Proof. (1) Let $E^{\prime}$ be the pullback of $E$ by $f$,

$$
E^{\prime}=\{(e, \gamma) \in E \times \Gamma: \pi(e)=f(\gamma)\}
$$

The diagram

$$
\begin{aligned}
& 0 \rightarrow A \rightarrow E^{\prime} \rightarrow \Gamma \rightarrow 0
\end{aligned}
$$

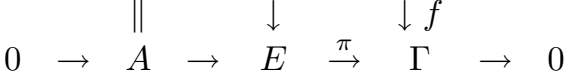

is exact and $f$ lifts to $\bar{f}: \Gamma \rightarrow E$ if and only if the top row in (3) splits. By Exercise 6.6.4 in We this happens if and only if $f^{*}(\xi)=0$. We get the second part of the statement of Lemma 12 by chasing the arrows of diagram (3).

Lemma 13. Let $g_{1}, \ldots, g_{n}$ be the free generators of $F_{n}$ and let $e_{1}, \ldots, e_{n}$ be the canonical basis of $\mathbb{Z}^{n}$. There exists an isomorphism $\kappa: \bigwedge^{2} \mathbb{Z}^{n} \rightarrow F_{n, 1} / F_{n, 2}$ such that

$$
\kappa\left(e_{i} \wedge e_{j}\right)=\left[g_{i}, g_{j}\right] .
$$

Proof. Since $F_{n, 1} / F_{n, 2}$ is abelian and $\bigwedge^{2} \mathbb{Z}^{n}$ is a free abelian group with basis $e_{i} \wedge e_{j}, i<j$, there is unique homomorphism $\kappa: \bigwedge^{2} \mathbb{Z}^{n} \rightarrow F_{n, 1} / F_{n, 2}$ defined by (4) for $i<j$. Now note that (4) holds for all $i, j$ and that $\kappa$ is an epimorphism. Since, by Corollary 5.12(iv) and Theorem 5.11 in [MKS] $F_{n, 1} / F_{n, 2}$ is a free abelian group of the same rank as $\bigwedge^{2} \mathbb{Z}^{n}, \kappa$ is an isomorphism.

Theorem 14. $d_{2}(\Gamma)=c_{2}(\Gamma)$ for any group $\Gamma$.

Proof. If $c_{2}(\Gamma)=n$, then there exists an epimorphism $f=\left(f_{1}, \ldots, f_{n}\right): \Gamma \rightarrow \mathbb{Z}^{n}$ such that $f_{i} \cup f_{j}=0$ in $H^{2}(\Gamma, \mathbb{Z})$ for any $i, j$. Consider the central extension

$$
0 \rightarrow \bigwedge^{2} \mathbb{Z}^{n}=F_{n, 1} / F_{n, 2} \rightarrow F_{n} / F_{n, 2} \stackrel{\pi}{\rightarrow} F_{n} / F_{n, 1}=\mathbb{Z}^{n} \rightarrow 0 .
$$

We will show that $f$ lifts to an epimorphism $\bar{f}: \Gamma \rightarrow F_{n} / F_{n, 2}$ such that $f=\pi \bar{f}$.

\footnotetext{
${ }^{6}$ This statement does not follow directly from the definition of $c_{k}(X)$ since the cohomology classes in Definition 5 are not assumed to be primitive.
} 
Since $F_{n, 1} / F_{n, 2}$ lies in the center of $F_{n} / F_{n, 2}$, the above extension corresponds to $\xi \in H^{2}\left(\mathbb{Z}^{n}, \bigwedge^{2} \mathbb{Z}^{n}\right)$, where the action of $\mathbb{Z}^{n}$ on $\bigwedge^{2} \mathbb{Z}^{n}$ is trivial. Consider the function $\sigma: \mathbb{Z}^{n} \rightarrow F_{n} / F_{n, 2}, \sigma\left(\sum_{i=1}^{n} a_{n} e_{n}\right)=\prod_{i=1}^{n} g_{i}^{a_{i}}$. The cohomology class $\xi$ is represented by the cocycle $\xi\left(v_{1}, v_{2}\right)=\sigma\left(v_{1}\right) \sigma\left(v_{2}\right) \sigma\left(v_{1} v_{2}\right)^{-1}$; compare [We, §6.6]. We have $\xi\left(e_{i}, e_{j}\right)=1$ for $i \leq j$ and $\xi\left(e_{i}, e_{j}\right)=\left[g_{i}, g_{j}\right]$ for $i>j$. Hence, using the identities of [MKS, Thm 5.3] and the isomorphism $\kappa: \bigwedge^{2} \mathbb{Z}^{n} \rightarrow F_{n, 1} / F_{n, 2}$, we see that $\xi$ corresponds to the cocycle

$$
\xi\left(\sum a_{i} e_{i}, \sum b_{i} e_{i}\right)=\sum_{i>j} a_{i} b_{j} e_{i} \wedge e_{j}
$$

in $H^{2}\left(\mathbb{Z}^{n}, \bigwedge^{2} \mathbb{Z}^{n}\right)$. Hence, for $\gamma_{1}, \gamma_{2} \in \Gamma$,

$$
f^{*}(\xi)\left(\gamma_{1}, \gamma_{2}\right)=\sum_{i>j} f_{i}\left(\gamma_{1}\right) f_{j}\left(\gamma_{2}\right) e_{i} \wedge e_{j}=-\sum_{i>j}\left(f_{i} \cup f_{j}\right)\left(\gamma_{1}, \gamma_{2}\right) e_{i} \wedge e_{j} ;
$$

compare Bro, V§3]. Since $f_{i} \cup f_{j}=0$ in $H^{2}(\Gamma, \mathbb{Z})$, we also have $\xi=0$ in $H^{2}\left(\Gamma, \bigwedge^{2} \mathbb{Z}^{n}\right)$. Now, by Lemma 12] $f$ extends to an epimorphism $\bar{f}: \Gamma \rightarrow F_{n} / F_{n, 2}$.

\section{Cut numbers and Lie algebras}

In this section we prove Theorem 1, The background information about semisimple Lie algebras can be found in [Hu, Sa]. For any Lie algebra $\mathfrak{g}$ let $\Psi_{\mathfrak{g}}(x, y, z)=$ $\kappa(x,[y, z])$, for $x, y, z \in \mathfrak{g}$, where $\kappa$ is the Killing form on $\mathfrak{g}$. The form $\Psi_{\mathfrak{g}}$ is skewsymmetric.

For any closed, oriented 3-manifold $M$, let $\Psi_{M}: \bigwedge^{3} H^{1}(M, \mathbb{Q}) \rightarrow \mathbb{Q}, \Psi_{M}(x, y, z)$ $=(x \cup y \cup z)[M]$. Whenever convenient, we will assume that $\Psi_{M}$ is a 3 -form defined on $H^{1}(M, \mathbb{R})$.

Proposition 15. Let $M$ be a 3-manifold and let $\mathfrak{g}$ be a semisimple Lie algebra of compact type over $\mathbb{R}$. If there are isomorphisms of vector spaces $H^{1}(M, \mathbb{R}) \simeq \mathbb{R}^{n} \simeq \mathfrak{g}$ such that $\Psi_{M}=\Psi_{\mathfrak{g}}: \bigwedge^{3} \mathbb{R}^{n} \rightarrow \mathbb{R}$, then $b_{1}(M)=\operatorname{dim} \mathfrak{g}$ and $c_{2}(M) \leq \operatorname{rank} \mathfrak{g}$.

Proof. The equality $b_{1}(M)=\operatorname{dim} \mathfrak{g}$ is obvious. If $c_{2}(M)=k$, then there exist linearly independent vectors $v_{1}, \ldots, v_{k} \in H^{1}(M, \mathbb{R})$ such that $\Psi_{M}\left(v_{i}, v_{j}, H^{1}(M, \mathbb{R})\right)=$ 0 , for any $i, j$. Hence, under the identification $H^{1}(M, \mathbb{R}) \simeq \mathfrak{g}$, the vectors $v_{1}, \ldots, v_{k}$ span a $k$-dimensional abelian subalgebra of $\mathfrak{g}$. Since $\mathfrak{g}$ is of compact type, any two maximal abelian Lie subalgebras of $\mathfrak{g}$ are conjugate and their dimensions are equal to rank $g$. Therefore $k \leq \operatorname{rank} \mathfrak{g}$.

Now we are ready to prove Theorem 1 For any complex semisimple Lie algebra $\mathfrak{g}$ consider its Chevalley's basis $X_{\alpha}, H_{\alpha}$, where $\alpha$ 's are positive roots of $\mathfrak{g}$. The Lie algebra $\mathfrak{g}_{0}$ spanned by $i H_{\alpha}, X_{\alpha}-X_{-\alpha}, i\left(X_{\alpha}+X_{-\alpha}\right)$ is a real compact form of $\mathfrak{g}$; compare [Sa, Ch. I§10]. Furthermore, since in Chevalley's basis $\mathfrak{g}$ has integral structural constants, $\Psi_{\mathfrak{g}_{0}}$ is also defined over $\mathbb{Z}$. Hence $\Psi_{\mathfrak{g}_{0}}: \bigwedge^{3} \mathbb{Q}^{n} \rightarrow \mathbb{Q}$. By Sullivan's theorem $(\underline{\mathrm{Su}}])$ there exists a 3-manifold $M$ with $H^{1}(M, \mathbb{Q}) \simeq \mathbb{Q}^{n}$ and $\Psi_{M}=\Psi_{\mathfrak{g}_{0}}: \bigwedge^{3} \mathbb{Q}^{n} \rightarrow \mathbb{Q}$.

For any $\mathfrak{g}$, a manifold $M$ as above can be explicitly constructed using the method of Sullivan, $\underline{\mathrm{Su}}$. 


\section{INTERMEZZO: ClassifiCATION OF SKEW-SYMMETRIC FORMS}

Let $\mathbb{K}$ be a field of characteristic $\chi(\mathbb{K}) \neq 2$. For any $\Psi: \bigwedge^{s} \mathbb{K}^{n} \rightarrow \mathbb{K}^{m}$ we call the $k$-nullity of $\Psi, \operatorname{null}_{k}(\Psi)$, the maximum of dimensions of vector subspaces $W \subset \mathbb{K}^{n}$ such that

$$
\Psi(\underbrace{W, \ldots, W}_{k}, \underbrace{\mathbb{K}^{n}, \ldots, \mathbb{K}^{n}}_{s-k})=0 .
$$

$n-\operatorname{null}_{1}(\Psi)$ is called the rank of $\Psi$, and it is equal to the minimum of dimensions of spaces $V$ such that $\Psi$ factors through $\bigwedge^{s} V$. We will call $n u l l_{2}(\Psi)$ the nullity of $\Psi$.

The natural $G L_{n}(\mathbb{K})$-action on $\mathbb{K}^{n}$ yields a $G L_{n}(\mathbb{K})$-action on forms $\bigwedge^{s} \mathbb{K}^{n} \rightarrow$ $\mathbb{K}^{m}, g f\left(x_{1}, \ldots, x_{n}\right)=f\left(g^{-1} x_{1}, \ldots, g^{-1} x_{n}\right)$ for $g \in G L_{n}(\mathbb{K}), x_{1}, \ldots, x_{n} \in \mathbb{K}$. We say that $\Psi, \Psi^{\prime}: \bigwedge^{s} \mathbb{K}^{n} \rightarrow \mathbb{K}^{m}$ are isomorphic if they belong to the same orbit of the $G L_{n}(\mathbb{K})$-action.

The argument used in the previous section implies the following

Fact 16. For any $\Psi: \bigwedge^{3} \mathbb{Q}^{n} \rightarrow \mathbb{Q}$ with nullity $c$ there exists a 3-manifold $M$ with $b_{1}(M)=n$ and with $c_{2}(M) \leq c$.

Therefore, one is interested in constructing 3-forms of small nullity. This simplelooking problem is not easy to approach by any straightforward method. A part of the problem is that 3-forms do not admit classification similar to that for 2-forms: each skew-symmetric 2-form is uniquely determined (up to isomorphism) by its rank. More precisely, for any $\Phi: \bigwedge^{2} \mathbb{K}^{n} \rightarrow \mathbb{K}$ of rank $r$ there exists a basis $e_{1}, \ldots, e_{n}$ of $\mathbb{K}^{n}$ such that $\Phi$ in the dual basis is given by

$$
\Phi=e^{1} \wedge e^{r+1}+\ldots+e^{r} \wedge e^{2 r} .
$$

In contrast to 2 -forms, 3 -forms are not classified by their ranks. The classification of 3-forms of rank at most 8 over algebraically closed fields can be found in [Gu, §35]. There are no 3 -forms of rank 1, 2, 4. There is only one 3-form (up to an isomorphism) of rank $3, e^{1} \wedge e^{2} \wedge e^{3}$, and only one 3 -form of $\operatorname{rank} 5, e^{1} \wedge e^{2} \wedge e^{3}+e^{1} \wedge e^{2} \wedge e^{4}$. There are two types of forms of rank 6 :

$$
e^{1} \wedge e^{2} \wedge e^{3}+e^{4} \wedge e^{5} \wedge e^{6} \text { and } e^{1} \wedge e^{2} \wedge e^{3}+e^{3} \wedge e^{4} \wedge e^{5}+e^{2} \wedge e^{5} \wedge e^{6}
$$

The classification of 3 -forms of rank 7,8 is more complicated. The classification of 3 -forms of rank 9 (over $\mathbb{C}$ only) was carried out in $[\mathrm{VE}]$. The classification of 3 -forms of rank larger than 9 is not known.

Proposition 17. For any infinite field $\mathbb{K}$ there are infinitely many non-isomorphic forms $\Psi: \bigwedge^{3} \mathbb{K}^{n} \rightarrow \mathbb{K}$ for $n \geq 9$.

Proof. Assume that $n \geq 9$ and that up to an isomorphism $\Psi_{1}, \ldots, \Psi_{k}$ are the only skew-symmetric 3 -forms on $\mathbb{K}^{n}$. Let $X$ be the algebraic closure of $\bigcup_{i} G L_{n}(\overline{\mathbb{K}}) \Psi_{i}$ in $\left(\bigwedge^{3} \overline{\mathbb{K}}\right)^{*}$. We have $\operatorname{dim} G L_{n}(\overline{\mathbb{K}})=n^{2}<\left(\begin{array}{c}n \\ 3\end{array}\right)=\operatorname{dim}\left(\bigwedge^{3} \overline{\mathbb{K}}\right)^{*}$ and, therefore, $\operatorname{dim} X<$ $\operatorname{dim}\left(\bigwedge^{3} \overline{\mathbb{K}}\right)^{*}$. By Lemma $\mathbb{1 8}(2)$ (applied to $\left.U=\left(\bigwedge^{3} \overline{\mathbb{K}}\right)^{*} \backslash X\right)$ there exists $\Psi \in$ $\left(\bigwedge^{3} \mathbb{K}\right)^{*}$ such that $\Psi \notin X$. This contradicts the initial assumption.

Lemma 18. Let $\mathbb{K}$ be an infinite field. (1) For any non-zero $f \in \overline{\mathbb{K}}\left[x_{1}, \ldots, x_{n}\right]$ there exist infinitely many points $\left(a_{1}, \ldots, a_{n}\right) \in \mathbb{K}^{n}$ such that $f\left(a_{1}, \ldots, a_{n}\right) \neq 0$.

(2) If $U$ is a (Zariski) open subset of $\overline{\mathbb{K}}^{n}$, then there are infinitely many points in $U$ defined over $\mathbb{K}$ (i.e. with all their coordinates in $\mathbb{K}$ ). 
Proof. We prove (1) by induction. Assume that the statement holds for $n-1$. Any non-zero $f \in \overline{\mathbb{K}}\left[x_{1}, \ldots, x_{n}\right]$ is either constant (and then the statement of the lemma holds) or it has a positive degree with respect to at least one variable, say $x_{1}$. Therefore $f=\sum x_{1}^{i} f_{i}$, where $f_{i} \in \overline{\mathbb{K}}\left[x_{2}, \ldots, x_{n}\right]$ and $f_{k} \neq 0$ for at least one $k>0$. By our assumption there exist $a_{2}, \ldots, a_{n} \in \mathbb{K}$ such that $f\left(a_{2}, \ldots, a_{n}\right) \neq 0$ and hence, $f\left(\cdot, a_{2}, \ldots, a_{n}\right)$ is a non-zero polynomial in $\overline{\mathbb{K}}\left[x_{1}\right]$. Since $f\left(\cdot, a_{2}, \ldots, a_{n}\right)$ has only finitely many zeros, $f\left(a_{1}, a_{2}, \ldots, a_{n}\right) \neq 0$ for infinitely many $a_{1} \in \mathbb{K}$.

(2) Let $f \in \overline{\mathbb{K}}\left[x_{1}, \ldots, x_{n}\right]$ be one of the defining polynomials of $X=\overline{\mathbb{K}}^{n} \backslash U$, i.e. let the zero set of $f$ contain $X$. By (1) there are infinitely many points $\left(a_{1}, \ldots, a_{n}\right) \in \mathbb{K}^{n}$ such that $f\left(a_{1}, \ldots, a_{n}\right) \neq 0$. Each of these points lies in $U$.

The difficulties in understanding 3-forms explain the reason for using semisimple Lie algebras to construct 3 -forms of small nullity in the previous section.

\section{Nullity OF SKEW-SYMmetriC FORMS}

Since skew-symmetric forms cannot be classified directly, in this section we study their properties in more subtle ways. Our goal is proving that if $\mathbb{K}$ is an algebraically closed field, then (a) for any $n$ there exists $\Psi: \bigwedge^{3} \mathbb{K}^{n} \rightarrow \mathbb{K}$ with $\operatorname{null}(\Psi) \leq 2$ and (b) for any $k$ the set of forms $\Psi: \bigwedge^{3} \mathbb{K}^{n} \rightarrow \mathbb{K}$ with $\operatorname{null}(\Psi) \geq k$ forms a closed algebraic subset of the space of all 3 -forms. In the next section we will use these two results to prove Theorem 2 .

Since our methods of proof are not specifically limited to 3-forms, we formulate our results more broadly in terms of arbitrary skew-symmetric forms, hoping that one day someone will find new applications, unrelated to cut numbers of 3manifolds.

We start with the following result due to T. Goodwillie.

Theorem 19. Let $\mathbb{K}$ be algebraically closed.

(1) If $m \leq 2 n-4$, then the nullity of any form $\Phi: \bigwedge^{2} \mathbb{K}^{n} \rightarrow \mathbb{K}^{m}$ is at least 2 .

(2) If $m>2 n-4$, then there exists $\Phi: \bigwedge^{2} \mathbb{K}^{n} \rightarrow \mathbb{K}^{m}$ with null $(\Phi)=1$.

Proof. Consider the Grassmannian of 2-planes in $\mathbb{K}^{n}, G_{2}\left(\mathbb{K}^{n}\right)$, embedded into $\mathbb{P}\left(\bigwedge^{2} \mathbb{K}^{n}\right)$ by the map $V^{2} \rightarrow V^{2} \wedge V^{2}$. Observe that $\operatorname{null}(\Phi) \geq 2$ if and only if $\mathbb{P}(\operatorname{Ker} \Phi) \cap G_{2}\left(\mathbb{K}^{n}\right) \neq \emptyset$ in $\mathbb{P}\left(\bigwedge^{2} \mathbb{K}^{n}\right)$. A projective subvariety of dimension $s$ of $\mathbb{P}\left(\mathbb{K}^{N}\right)$ intersects every projective subspace of codimension at most $s$ in $\mathbb{P}\left(\mathbb{K}^{N}\right)$, but it does not intersect some projective subspaces of codimension $s+1$; compare $\mathrm{Ha}$. Thm I.7.2]. Since $\operatorname{dim} G_{2}\left(\mathbb{K}^{n}\right)=2 n-4$ and $\operatorname{codim} P(\operatorname{Ker} \Phi)=m, \operatorname{null}(\Phi) \geq 2$ for all $\Phi: \bigwedge^{2} \mathbb{K}^{n} \rightarrow \mathbb{K}^{m}$ with $m \leq 2 n-4$.

If $m \geq 2 n-4$, then there exists a plane $W \subset \wedge^{2} \mathbb{K}^{n}$ of codimension $m$ such that $\mathbb{P}(W) \cap G_{2}\left(\mathbb{K}^{n}\right)=\emptyset$. For any such $W$, the 2 -form $\Phi: \bigwedge^{2} \mathbb{K}^{n} \rightarrow \bigwedge^{2} \mathbb{K}^{n} / W=\mathbb{K}^{m}$ has nullity 1 .

By considering a 3 -form $\Psi: \bigwedge^{3} \mathbb{K}^{n} \rightarrow \mathbb{K}$ as a 2 -form, $\bigwedge^{2} \mathbb{K}^{n} \rightarrow\left(\mathbb{K}^{n}\right)^{*}$ we get the following corollary:

Corollary 20. For $n \geq 4$ the nullity of every $\Psi: \bigwedge^{3} \mathbb{K}^{n} \rightarrow \mathbb{K}$ is at least 2 .

Theorem 19 does not hold over fields which are not algebraically closed. Nevertheless, it is very possible that the above corollary holds over any field $\mathbb{K}$ of characteristic $\chi(\mathbb{K}) \neq 2$. 
Conjecture. For any field $\mathbb{K}$ of characteristic $\chi(\mathbb{K}) \neq 2$ and for $n \geq 4$, the nullity of every $\Psi: \bigwedge^{3} \mathbb{K}^{n} \rightarrow \mathbb{K}$ is at least 2 .

Proposition 21. The conjecture holds for even $n .7$

Proof. For any $v \in \mathbb{K}^{n}$ there is an $n \times n$ matrix $A(v)$ such that $\Psi\left(v, w_{1}, w_{2}\right)=$ $w_{1}^{T} \cdot A \cdot w_{2}$ for any $w_{1}, w_{2} \in \mathbb{K}^{n}$. Since $A \cdot v=0, \operatorname{rank} A \leq n-1$. Since $\chi(\mathbb{K}) \neq 2$ and $A$ is skew-symmetric, the null space of $A,\{w: A w=0\}$, has an even dimension and, hence, there is $w$ linearly independent from $v$ such that $\Psi\left(v, w, \mathbb{K}^{n}\right)=0$.

Theorem 22. Let $\mathbb{K}$ be an algebraically closed field. For any $s, k, r, n, m$, the set of forms $\Psi: \bigwedge^{s} \mathbb{K}^{n} \rightarrow \mathbb{K}^{m}$ with null $_{k}(\Psi) \geq r$ is a closed algebraic subset of $\left(\bigwedge^{s} \mathbb{K}^{n}\right)^{*} \otimes \mathbb{K}^{m}$ defined by homogeneous polynomials 8

Fix $s, k, r, n, m$. For each $r$-dimensional subspace $V \subset \mathbb{K}^{n}$ consider

$$
\imath(V)=\bigwedge^{k} V \wedge \bigwedge^{s-k} \mathbb{K}^{n}=\operatorname{Span}\left\{v_{1} \wedge \ldots \wedge v_{k} \wedge w_{1} \wedge \ldots \wedge w_{s-k}\right\} \subset \bigwedge^{s} \mathbb{K}^{n},
$$

where $v_{1}, \ldots, v_{k} \in V, w_{1}, \ldots, w_{s-k} \in \mathbb{K}^{n}$.

Lemma 23. For any $V$ as above, $\operatorname{dim} \imath(V)=d$, where

$$
d=\sum_{k \leq i \leq r}\left(\begin{array}{l}
i-1 \\
k-1
\end{array}\right)\left(\begin{array}{l}
n-i \\
s-k
\end{array}\right) .
$$

Proof. If $e_{1}, \ldots, e_{n}$ is a basis of $\mathbb{K}^{n}$ such that $V=\operatorname{Span}\left\{e_{1}, \ldots, e_{r}\right\}$, then $\imath(V)$ has a basis composed of elements $e_{i_{1}} \wedge \ldots \wedge e_{i_{k}} \wedge \ldots \wedge e_{i_{s}}$, where $1 \leq i_{1}<\ldots<i_{k}<\ldots<i_{s} \leq$ $n$ and $i_{k} \leq r$. The number of basis elements of $\imath(V)$ with $i_{k}=i$ is $\left(\begin{array}{c}i-1 \\ k-1\end{array}\right)\left(\begin{array}{l}n-i \\ s-k\end{array}\right)$.

Let

$$
X_{k, r, s, n}=\left\{\imath(V): V \in G_{r}\left(\mathbb{K}^{n}\right)\right\} \subset G_{d}\left(\bigwedge^{s} \mathbb{K}^{n}\right) .
$$

We have

$$
\operatorname{null}_{k}(\Psi) \geq r \text { if and only if } G_{d}(\operatorname{Ker} \Psi) \cap X_{k, r, s, n} \neq \emptyset \text { in } G_{d}\left(\bigwedge^{s} \mathbb{K}^{n}\right) .
$$

Proof of Theorem 20. The proof relies on elimination theory for projective spaces; see [CLO, Ch. 8§5]. (Compare also vW] $§ 80$, p.8]).

Let $\left\{e_{1}, \ldots, e_{N}\right\}$ be a basis of $\bigwedge^{s} \mathbb{K}^{n}, N=\left(\begin{array}{l}n \\ s\end{array}\right)$. Using this basis we identify $\bigwedge^{s} \mathbb{K}^{n}$ with $\mathbb{K}^{N}$. The Grassmannian $G_{d}\left(\mathbb{K}^{N}\right)$ embeds into a projective space by $P: G_{d}\left(\mathbb{K}^{N}\right) \hookrightarrow \mathbb{P}\left(\bigwedge^{d} \mathbb{K}^{N}\right), P(W)=\bigwedge^{d} W$. Each $P(W)$ can be written as

$$
\sum_{1 \leq i_{1}<\ldots<i_{d} \leq N} P_{i_{1} \ldots i_{d}}(W) e_{i_{1}} \wedge \ldots \wedge e_{i_{d}}
$$

where the numbers $P_{i_{1} \ldots i_{d}}(W)$, called the Plücker coordinates of $W$, are defined up to common scalar multiplication.

Let $\Psi: \mathbb{K}^{N} \rightarrow \mathbb{K}^{m}$ be given by as $\Psi(x)=\sum_{i=1}^{N} \Psi_{i} e^{i}(x)$, where $\left\{e^{1}, \ldots, e^{N}\right\}$ is the basis of $\left(\mathbb{K}^{N}\right)^{*}$ dual to $\left\{e_{1}, \ldots, e_{N}\right\}$ and $\Psi_{i}=\left(\Psi_{i j}\right)_{1 \leq j \leq m} \in \mathbb{K}^{m}$.

Let $M_{k l}(\mathbb{K})$ denote the set of $k \times l$ matrices with entries in $\mathbb{K}$.

\footnotetext{
${ }^{7}$ We can also prove the conjecture for $n=5$.

${ }^{8}$ Recall that $\operatorname{null}_{k}(\Psi)$ was defined in Section 4 .
} 
Lemma 24. There exist polynomial functions $F_{1}, \ldots, F_{f}: \bigwedge^{d} \mathbb{K}^{N} \times M_{N, m}(\mathbb{K}) \rightarrow \mathbb{K}$ such that for any $\Psi=\sum_{i=1}^{N} \Psi_{i} e^{i}$, the vector $\sum_{1 \leq i_{1}<\ldots<i_{d} \leq N} P_{i_{1} \ldots i_{d}} e_{i_{1}} \wedge \ldots \wedge e_{i_{d}} \in$ $\bigwedge^{d} \mathbb{K}^{N}$ belongs to $\bigwedge^{d} \operatorname{Ker} \Psi$ if and only if

$$
F_{i}\left(\left(P_{i_{1} \ldots i_{d}}\right)_{1 \leq i_{1}<\ldots<i_{d} \leq N}, \Psi_{1}, \ldots, \Psi_{N}\right)=0 \text { for } 1 \leq i \leq f .
$$

Furthermore, the polynomial functions $F_{1}, \ldots, F_{f}$ are homogeneous with respect to the coordinates of $\bigwedge^{d} \mathbb{K}^{N}$.

Proof. Denote $\operatorname{dim} \operatorname{Ker} \Psi=N-m$ by $t$. Since the statement is obvious for $t<d$, assume that $t \geq d$. The vectors $v_{1}=\sum_{j=1}^{N} a_{1 j} e_{j}, \ldots, v_{t}=\sum_{j=1}^{N} a_{t j} e_{j}$ belong to $\operatorname{Ker} \Psi$ if and only if

$$
\sum_{j=1}^{N} a_{i j} \Psi_{j}=0 \text { in } \mathbb{K}^{m} \text { for every } 1 \leq i \leq t .
$$

Note that

$$
v_{i_{1}} \wedge \ldots \wedge v_{i_{d}}=\sum_{1 \leq j_{1}<\ldots<j_{d} \leq N} \operatorname{det}\left(\begin{array}{ccc}
a_{i_{1}, j_{1}} & \ldots & a_{i_{d}, j_{1}} \\
\ldots & \ldots & \ldots \\
a_{i_{1}, j_{d}} & \ldots & a_{i_{d}, j_{d}}
\end{array}\right) e_{j_{1}} \wedge \ldots \wedge e_{j_{d}} .
$$

Therefore, $w \in \bigwedge^{d} \mathbb{K}^{N}$ belongs to $\bigwedge^{d} \operatorname{Ker} \Psi$ if and only if there exist $\left(a_{i j}\right) \in M_{t, N}$ satisfying (6) and there exist $b_{i_{1} \ldots i_{d}} \in \mathbb{K}$ for all $1 \leq i_{1}<\ldots<i_{d} \leq t$ such that

$$
w=\sum_{\substack{1 \leq j_{1}<\ldots<j_{d} \leq N \\
1 \leq i_{1}<\ldots<i_{d} \leq t}} b_{i_{i} \ldots i_{d}} \operatorname{det}\left(\begin{array}{ccc}
a_{i_{1}, j_{1}} & \ldots & a_{i_{d}, j_{1}} \\
\ldots & \ldots & \ldots \\
a_{i_{1}, j_{d}} & \ldots & a_{i_{d}, j_{d}}
\end{array}\right) e_{j_{1}} \wedge \ldots \wedge e_{j_{d}} .
$$

Since (6) and (7) are homogeneous equations with respect to $\left(a_{i j}\right)$ and $\left(b_{i_{1} \ldots i_{d}}\right)_{1 \leq i_{1}<\ldots<i_{d} \leq t}$, by [CLO, Ch. $\left.8 \S 5 \mathrm{Thm} 8\right]$ there exist polynomial functions $H_{1}, \ldots H_{h}$ on $\bigwedge^{d} \mathbb{K}^{N} \times M_{N, m}(\mathbb{K})$ such that for any $\Psi_{1}, \ldots, \Psi_{N} \in \mathbb{K}^{m}$ the equations

$$
H_{i}\left(\cdot, \Psi_{1}, \ldots, \Psi_{N}\right)=0 \text { for } 1 \leq i \leq h
$$

describe the set $\bigwedge^{d} \operatorname{Ker} \Psi$. For each $i$ let $H_{i j}$ be the sum of monomials of $H_{i}$ of total degree $j$ with respect to the coordinates of $\bigwedge^{d} \mathbb{K}^{N}, H_{i}=\sum_{j} H_{i j}$. Since $\alpha \in$ $\bigwedge^{d} \operatorname{Ker} \Psi$ if and only if $c \alpha \in \bigwedge^{d} \operatorname{Ker} \Psi$, for any $c \in \mathbb{K}^{*}, H_{i j}\left(\alpha, \Psi_{1}, \ldots, \Psi_{N}\right)=0$ for any $\Psi_{1}, \ldots, \Psi_{N} \in \mathbb{K}^{m}$ and $\alpha \in \bigwedge^{d} \operatorname{Ker} \Psi$, for $\Psi=\sum \Psi_{i} e^{i}$. Hence the polynomials $H_{i j}$ satisfy the conditions of the lemma.

We continue the proof of Theorem 22, As before, let $\bigwedge^{s} \mathbb{K}^{n}=\mathbb{K}^{N}$ and consider the embedding $P: G_{d}\left(\mathbb{K}^{N}\right) \hookrightarrow \mathbb{P}\left(\bigwedge^{d} \mathbb{K}^{N}\right)$. Let $G_{1}, \ldots, G_{g}: \bigwedge^{d} \mathbb{K}^{N} \rightarrow \mathbb{K}$ be homogeneous polynomials describing $P\left(X_{k, r, s, n}\right) \subset \mathbb{P}\left(\bigwedge^{d} \mathbb{K}^{N}\right)$. By (5) null $l_{k}(\Psi) \geq r$ if and only if the polynomials $G_{i}(\cdot)$ and $F_{i}\left(\cdot, \Psi_{1}, \ldots, \Psi_{N}\right)$ (defined in Lemma 24) have a common solution in $\bigwedge^{d} \mathbb{K}^{N}$ different from 0. By [CLO, Ch. 8§5 Thm 8], the set of $\left(\Psi_{1}, \ldots, \Psi_{N}\right)$ for which such a solution exists is an algebraic set in $M_{N, m}(\mathbb{K})$. An argument analogous to that given at the end of proof of previous lemma shows that this set can be described by homogeneous polynomials. This completes the proof of Theorem 22,

Corollary 25. If $\mathbb{K}$ is an algebraically closed field, then for any $s, k, r, n, m$, there is an algorithm (using Gröbner bases) for calculating null $(\Psi)$ for $\Psi: \bigwedge^{s} \mathbb{K}^{n} \rightarrow \mathbb{K}^{m}$. 
Any $\Psi: \bigwedge^{s} \mathbb{K}^{n} \rightarrow \mathbb{K}^{m}$ yields $\bar{\Psi}: \bigwedge^{s} \overline{\mathbb{K}}^{n} \rightarrow \overline{\mathbb{K}}^{m}$ with $\operatorname{null}_{k}(\bar{\Psi}) \geq \operatorname{null}_{k}(\Psi)$ for any $k$.

For any field $\mathbb{K}$ the process of eliminating variables of polynomials over $\mathbb{K}$ produces polynomials over the same field $\mathbb{K}$. Therefore, the proof of Theorem 22 implies a more general statement:

Corollary 26. For an arbitrary field $\mathbb{K}$ and for any $s, k, r, n, m$, the set of forms $\Psi: \bigwedge^{s} \mathbb{K}^{n} \rightarrow \mathbb{K}^{m}$ with null $_{k}(\bar{\Psi}) \geq r$ is a closed algebraic subset of $\left(\bigwedge^{s} \mathbb{K}^{n}\right)^{*} \otimes \mathbb{K}^{m}$ defined by homogeneous polynomials with coefficients in $\mathbb{K}$.

Theorem 27. For any algebraically closed field $\mathbb{K}$ and for any $n$ there exists $\Psi$ : $\bigwedge^{3} \mathbb{K}^{n} \rightarrow \mathbb{K}$ with $\operatorname{null}_{2}(\Psi) \leq 2$.

Proof. Since for $n \leq 3$ the statement is obvious, assume that $n \geq 4$. If there exists a hyperplane $W \subset \bigwedge^{3} \mathbb{K}^{n}$ such that $G_{d}(W) \cap X_{k, r, s, n}=\emptyset$ for $k=2, r=s=3$, then by (5), the projection map $\Psi: \bigwedge^{3} \mathbb{K}^{n} \rightarrow \bigwedge^{3} \mathbb{K}^{n} / W=\mathbb{K}$ will be a skew-symmetric 3 -form on $\mathbb{K}^{n}$ with $\operatorname{null}_{2}(\Psi) \leq 2$. We are going to prove that such $W$ exists.

Let $W_{0}$ be any hyperplane in $\bigwedge^{3} \mathbb{K}^{n}$. Since the Grassmannian of $d$-dimensional planes in $\mathbb{K}^{N}$ has dimension $d N-d^{2}$ and $\operatorname{dim} X \leq \operatorname{dim} G_{3}\left(\mathbb{K}^{n}\right)$, we have

$$
\operatorname{dim} G_{d}\left(W_{0}\right)+\operatorname{dim} X \leq d\left(\left(\begin{array}{l}
n \\
3
\end{array}\right)-1\right)-d^{2}+3 n-9
$$

and

$$
\operatorname{dim} G_{d}\left(\bigwedge^{3} \mathbb{K}^{n}\right)=d\left(\begin{array}{l}
n \\
3
\end{array}\right)-d^{2}
$$

By Lemma 23 $d=3 n-8$ and, therefore,

$$
\operatorname{dim} G_{d}\left(W_{0}\right)+\operatorname{dim} X<\operatorname{dim} G_{d}\left(\bigwedge^{3} \mathbb{K}^{n}\right) .
$$

Since $G_{d}\left(\bigwedge^{3} \mathbb{K}^{n}\right)$ is a homogeneous $G L\left(\bigwedge^{3} \mathbb{K}^{n}\right)$-space, there exists $g \in G L\left(\bigwedge^{3} \mathbb{K}^{n}\right)$ such that $X$ and $g G_{d}\left(W_{0}\right)$ are transversal, i.e., $X \cap g G_{d}\left(W_{0}\right)=\emptyset$; compare [Ha, Thm III.10.8]. (Note that since $G_{d}\left(W_{0}\right)$ is smooth, Hartshorne's assumption that $\mathbb{K}$ has characteristic 0 is not necessary here.) Since $g G_{d}\left(W_{0}\right)=G_{d}\left(g W_{0}\right)$, the hyperplane $W=g W_{0}$ satisfies the required condition.

Note that we proved more:

Corollary 28. If $\mathbb{K}$ is algebraically closed and $s, k, r, n, m$ are such that $r \cdot n-r^{2}<$ $d \cdot m$, where $d$ is as in Lemma [23, then there exists a form $\Psi: \bigwedge^{s} \mathbb{K}^{n} \rightarrow \mathbb{K}^{m}$ with $\operatorname{null}_{k}(\Psi)<r$.

\section{Proof of Theorem 2}

(1) By Corollary [26, $X=\left\{\Psi: \operatorname{null}_{2}(\bar{\Psi})>2\right\}$ is a closed algebraic subset of $\left(\bigwedge^{3} \mathbb{Q}^{n}\right)^{*}$. By Theorem [27, $U=\left(\bigwedge^{3} \mathbb{Q}^{n}\right)^{*} \backslash X$ is a nonempty, and hence dense, open subset of $\left(\bigwedge^{3} \mathbb{Q}^{n}\right)^{*}$. Since $c(M) \leq \operatorname{null}_{2}\left(\Psi_{M}\right) \leq$ null $_{2}\left(\overline{\Psi_{M}}\right)$, the set of forms $\Psi_{M}$ for 3-manifolds $M$ with $c(M) \leq 2$ contains $U$.

(2) follows from (1).

The proof (3) is analogous to the proof of Proposition [17 Fix $n \geq 9$ and assume that the 3-manifolds $M$ with $b_{1}(M)=n$ and $c(M) \leq 2$ represent only finitely many different rational cohomology rings corresponding to $\Psi_{1}, \ldots, \Psi_{k}: \bigwedge^{3} \mathbb{Q}^{n} \rightarrow \mathbb{Q}$. Let $Y=\overline{\bigcup_{i} G L_{n}(\mathbb{C}) \Psi_{i}} \subset\left(\bigwedge^{3} \mathbb{C}^{n}\right)^{*}$. Since the set $X$ (defined above) and the set $Y$ 
are closed and strictly contained in $\left(\bigwedge^{3} \mathbb{C}^{n}\right)^{*}$, by Lemma 18(2) there exists $\Psi$ : $\bigwedge^{3} \mathbb{Q}^{n} \rightarrow \mathbb{Q}$ such that $\Psi \notin X \cup Y$. A contradiction.

\section{ACKNOWLEDGMENTS}

We thank S. Boyer, T. Kerler, T. Goodwillie, J. Millson, J. H. Przytycki, J. Roberts, A. Razborov, G. Schwartz, J. Stallings, and V. Turaev for their helpful comments.

\section{REFERENCES}

[Bre] G. E. Bredon, Topology and Geometry, Graduate Texts in Mathematics, Springer-Verlag, 1995.

[Bro] K. S. Brown, Cohomology of groups, Graduate Texts in Mathematics, Springer-Verlag, 1982. MR83k:20002

[CLO] D. Cox, J. Little, D. O'Shea, Ideals, Varieties, and Algorithms, An Introduction to Computational Algebraic Geometry and Commutative Algebra, 2nd edition, Springer, 1997. MR97h:13024

[CM] T. D. Cochran, P. Melvin, Quantum cyclotomic orders of 3-manifolds, Topology 40 (2001), no. 1, 95-125. MR2002f:57022

[Dw] W. G. Dwyer, Homology, Massey products and maps between groups, J. Pure Appl. Alg 6 (1975), 177-190. MR52:6710

[Fe] R. A. Fenn, Techniques of Geometric Topology, Cambridge Univ. Press, 1983. MR87a:57002

[Gi] P. Gilmer, Integrality for TQFTs, preprint, www.arxiv.org/abs/math.QA/0105059, to appear in Duke Math. Journal.

[Gu] G. B. Gurevich, Foundations of the theory of algebraic invariants, P. Noordhoff Ltd Groningen, The Netherlands, 1964. MR32:1211

[Ha] R. Hartshorne, Algebraic Geometry, Graduate Texts in Mathematics, Springer-Verlag, 1977. MR 57:3116

[Har] S. Harvey, On the Cut Number of a 3-manifold, Geom. Topol. 6 (2002) 409-424. MR2003g:57017

[He] J. Hempel, 3-manifolds, Annals of Mathematical Studies 86, Princeton Univ. Press, 1976. MR 54:3702

[Hu] J. E. Humphreys, Introduction to Lie Algebras and Representation Theory, Graduate Texts in Mathematics, Springer-Verlag, 1972. MR 48:2197

[LR] C. J. Leininger, A. W. Reid, The co-rank conjecture for 3-manifold groups, Algebr. Geom. Topol. 2 (2002), 949-1000. MF:2002m:57019

[MKS] W. Magnus, A. Karrass, D. Solitar, Combinatorial Group Theory, Dover Publications, Inc. 1976. MR.54:10423

[Ra] A. A. Razborov, On systems of equations in a free group, Izv. Akad. Nauk SSSR Ser. Mat. 48 (1984), no. 4, 779-832. English translation: Math. USSR Izvestiya 25 (1985), no. 1, 115-162. MF 86c:20033

[Sa] H. Samelson, Notes on Lie Algebras, Van Nostrand Reinhold Co., 1969. MR 40:7322

[St] J. R. Stallings, Problems about free quotients of groups, Geometric group theory (Columbus, OH, 1992), 165-182, Ohio State Univ. Math. Res. Inst. Publ. 3, de Gruyter, Berlin, 1995. MR97b:20028

[Su] D. Sullivan, On the intersection ring of compact three manifolds, Topology 14 (1975), 275-277. Mr.52:4296

[T1] V. Turaev, Milnor invariants and Massey products, J. Soviet Math. 12 (1979), 128-137.

[T2] V. Turaev, Cohomology rings, linking forms and invariants of spin structures of threedimensional manifolds, Mat. Sb. (N.S.) 120 (162) (1983), no. 1, 68-83, 143. English translation from: Math. USSR Sbornik 48 no 1 (1984), 65-79. MR84g:57009

[vW] B. L. van der Waerden, Modern Algebra, Frederick Ungar Pub. Co., vol II, 1950. 
[VE] E. B. Vinberg, A. G. Èlashvili, Classification of Trivectors of a 9-Dimensional Space, Trudy Sem. Vector. Tenzor. Anal. 18 (1978), 197-233. English translation: Sel. Math. Sov. 7 No 1 (1988) 63-98. MR80b:15039

[We] C. A. Weibel, An Introduction to Homological Algebra, Cambridge Univ. Press, 1994. MR95f:18001

Department of Mathematics, 244 Mathematics Building, Suny at Buffalo, Buffalo, NEW YorK 14260

E-mail address: asikora@buffalo.edu 